\title{
A Comparison of Seven Methods for Obtaining Subjective Descriptions of Judgmental Policy ${ }^{1}$
}

\author{
Richard L. Cook ${ }^{2}$ and Thomas R. Stewart \\ Institute of Behavioral Science, University of Colorado
}

\begin{abstract}
When subjective descriptions of judgmental policies have been compared to statistical descriptions of policies, investigators have repeatedly found an imperfect correspondence between subjective and statistical measures of importance. There is reason to believe, however, that these findings may be due to the use of inadequate procedures for obtaining subjective weights. The present study, therefore, compared seven methods for obtaining subjective descriptions of cue importance. Subjective policy descriptions were found to correspond reasonably well to statistical policy descriptions for both a three-cue and a seven-cue task, but the seven methods did not differ. All methods yielded more accurate predictions of actual judgments than an arbitrary policy of equal weights on all cues.
\end{abstract}

Most judgment tasks require a decision maker to integrate information from several sources to arrive at a single judgment about an environmental criterion. Hammond and Summers (1972) have reviewed research with multiple-cue tasks and concluded that a subject's achievement in such situations depends on both knowledge about a valid policy for integrating task information and control of the application of that policy.

There is ample evidence that the latter objective, reliable application or control of a judgmental policy, is difficult for many decision makers to attain (Goldberg, 1970, 1971; Dudycha \& Naylor, 1966; Hoffman \& Blanchard, 1961). But there has been little research directed at establishing how well a decision maker understands the policy he is using.

\section{Estimates of Subjective Weights}

The correspondence between subjective estimates of the relative influence of different cues on judgments and statistical weights yielded by

${ }^{1}$ This paper is based on research undertaken at the Institute of Behavioral Science, University of Colorado, and is Publication 158 of the Institute. The research was supported by National Institute of Mental Health Grant MH-16437. We thank David A. Summers for his advice about the design of the study, Kenneth $R$. Hammond for helpful comments on the paper, and Karene Will and Bertha Ramsay for their assistance with the analyses.

${ }^{2}$ Requests for reprints should be sent to Richard L. Cook, Institute of Behavioral Science, University of Colorado, Boulder, CO 80302. 
multiple regression procedures has been the object of several studies (see Slovic \& Lichtenstein (1971) for a review). The most frequently used procedure for assessing subjective weights has been to have judges distribute 100 points across the cues so as to reflect the role each cue played in their decisions (Hoffman, 1960).

The general conclusion reached by Slovic and Lichtenstein is that judges do not describe their weights very accurately; there is a consistent tendency to overestimate the number of cues that are important. If subjective weights are compared to regression weights, the subjects tend to overestimate the weights of unimportant cues and to underestimate the weights of important cues (see Slovic, 1969).

An objective method for examining correspondence between subjective and statistical weights is to correlate the two sets of weights. Although such correlations tend to be low (Slovic, Fleissner \& Bauman, 1972; Slovic, 1969; Hoffman, 1960; Permut, 1973), the meaningfulness of these correlations is questionable. Use of the subjective weights in a linear prediction model might yield predictions which differed little from those based on the optimal statistical weights, even though the subjective weights were not highly correlated with the statistical weights. If the correlation between statistical and subjective weights is the criterion, then one might conclude that a person's subjective weights were "wrong" when actually they were nearly as good as statistical weights for predicting actual judgments.

Summers, Taliaferro, and Fletcher (1970) used a different method for measuring the accuracy of subjective weights. They compared predictions of judgments based on subjective weights with actual judgments. Undergraduate subjects made a series of judgments for a four-cue task and used the method of dividing 100 points to indicate subjective weights for the cues. The subjective weights were used as if they were regression weights to calculate predicted judgments. The median correlation between predicted and actual judgments was $.60\left(r^{2}=.36\right)$; the median multiple correlation coefficient based on objective weights, however, was $.75\left(R^{2}=.56\right)$. Therefore, the use of subjective weights accounted for $20 \%$ less variance in the subjects' judgments than use of optimal linear statistical weights.

The above studies have each compared a single method for obtaining subjective weights with a single set of statistical weights. In contrast to this approach, Blood (1971) examined the usefulness of three different statistical concepts of weight: (a) multiple correlation beta weights, (b) zero-order correlation coefficients, and (c) the contribution of a predictor to the squared multiple correlation (Darlington, 1968). The data were subjective ratings made by clerical workers of each of five aspects of job satisfaction and ratings of overall job satisfaction. None of the 
alternative statistical weights corresponded closely to rankings of the importance of the five job aspects that had also been obtained from the workers.

While the above studies suggest that subjective reports of judgmental policies are not very accurate when compared with linear regression weights or with the variance accounted for in the subjects' own judgments by a linear model, the effect of the method used to assess subjective policies has not been systematically studied. Thus, while the method of asking subjects to divide 100 points is intuitively appealing, its actual effectiveness has not been examined. Other scaling procedures (Torgerson, 1958) might yield better estimates of subjective weights. And if some decision makers are using cues in a nonlinear or configural manner (Einhorn, 1970, 1971) more complex scaling procedures will be necessary.

\section{The Present Study}

The present study addresses the following questions.

1. Which methods for obtaining estimates of subjective weights are most accurate?

2. Are methods that allow a decision maker to indicate nonlinear cue utilization more accurate than simpler methods that yield only an estimate of overall cue weights?

3. Do task differences influence the accuracy of subjective estimates of judgmental policy?

The performance criterion chosen was the one suggested by Summers et al.: the correlation between actual ratings and predicted ratings derived from subjective weights. This criterion was chosen because it is both intuitively appealing and theoretically significant. It is intuitively appealing because it answers the question "How well does it work?" If, for a given task, the subjective weights yield predicted judgments correlating highly with actual judgments, then that set of subjective weights would seem to be a satisfactory description of the decision maker's policy even if the correlation between objective and subjective weights is low.

The procedure is also related to the theoretical developments of Hammond and Summers (1972) referred to earlier. The correlation between actual judgments and judgments predicted from subjective weights is comparable to their measure of achievement where the comparison policy is perfectly predictable by a linear rule; the multiple correlation coeffieient for the subject's judgments is a measure of his control over the use of his policy; and the ratio of the first correlation to the multiple correlation is equivalent to the measure of knowledge discussed by Ham- 
mond and Summers. This ratio will be less than 1.0 if the judge does not completely understand and consistently apply his policy or if the scaling method does not allow him to specify the policy accurately.

\section{METHOD}

Subjects

A questionnaire was distributed to faculty and graduate student members of the College of Arts and Sciences at the University of Colorado. Departments for which the Graduate Record Examination was not an entrance requirement or which had no graduate program were excluded. There were 143 complete responses, representing a rate of return of about $10 \%$. The number of respondents to each form of the questionnaire is included in Table 1 . Of the 143 responses, 71 were from faculty members and 72 from graduate students.

\section{Procedure}

All judges were presented both three-cue and seven-cue tasks consisting of a series of 55 profiles of graduate students applying for financial aid and 55 profiles of applicants to graduate school. Although judges were to assume that the profiles were of students either studying in or applying for admission to the judges' own departments, the profiles were actually of hypothetical candidates. Values for the cues were generated so that the cues were essentially uncorrelated $(|\bar{r}|=.10)$ and distributed rectangularly. The first five profiles served to allow the judges to adapt to the requirements of the task and only judgments based on the final 50 profiles are considered in the following analyses. Order of presentation of the two tasks was randomized.

A seven-point rating scale was used for both tasks, with a rating of 7 indicating a candidate the judge thought to be highly qualified for financial aid or admission to the department, a rating of 1 indicating a candidate who was not at all qualified, and a rating of 4 describing a candidate the judge felt had average qualifications.

Financial aid ratings were to be based on three cues: need, grade point average (GPA), and a faculty rating. The five levels of need were verbally described and ranged from no need (independent sources of income) to extreme need (could not continue without aid). The GPA referred to graduate courses and could range from 3.00-4.00. Five levels were possible for a faculty estimate of the applicant's promise for future success in his field, ranging from doubtful promise to excellent promise.

Graduate admissions ratings were based on seven cues. Three GRE scores were given: verbal, quantitative, and the advanced test in the 
candidate's field, the scores ranging from 400-750. Both an overall GPA, ranging from 2.75-4.00, and a major field GPA, ranging from 3.00-4.00, were given and assumed to be based on $4 \mathrm{yr}$ of undergraduate work. The favorability of letters from the references of each candidate was described by one of five verbal labels, with "outstanding prospect" and "express reservations" as the extremes. Five different verbal labels were also used to describe the quality of the undergraduate institution of the candidate, and these could vary from "well above average" to "well below average."

The instructions emphasized that the cases presented included only a selected sample of the information available from the record of each candidate and asked that the judges accept this limitation and make their evaluations on the basis of the information given. We chose cues that seemed to result in plausible abstractions of these decision problems.

Each judge also used one of seven methods to indicate his subjective estimates of the weights he had placed on each of the cues in making the financial aid and graduate admissions ratings. Each judge used the same method for both of the tasks, and the estimates were made when each task was completed.

\section{Subjective Rating Methods}

1. Dividing 100 points. The judge was to rate each of the three (or seven) cues so that a high rating represented a relatively important cue and the sum of the ratings equaled 100 .

2. Rating on a 100-point scale. As above, important information would receive a high rating, but there was no restriction on the sum of the ratings.

3. Paired comparisons. Each cue was paired with every other cue in each task. The judge indicated that the cues were of equal importance (0), or that one cue was slightly (1), substantially (2), or much more (3) important than the other. The number in parentheses was assigned to the more important cue and the weight for each cue was taken as the sum of these values for all comparisons in which the cue appeared.

4. Ratio. The judge chose one cue that he felt was of moderate importance to be used as a standard. This cue was given a rating of 100 points. Judges were instructed to rate the other cues so that the ratio of the ratings reflected relative importance of the cues.

5. Times influential. The judge indicated the number of times, out of a possible 55, that a given cue was "influential" in his decisions.

6. Individual cue ratings $I$. Judges indicated their "most likely" judgments for values of each cue taken separately, as if this were the only information available for a candidate. Numerical cues were divided into 
five (GPA's) or six (GRE's) intervals. Thus, for example, a judge might give a graduate school applicant a rating of 5 if he knew only that the applicant's major field GPA was 3.6-3.8. These judges also used the first rating method (dividing 100 points) to estimate the overall weight given to each cue.

7. Individual cue ratings $I I$. Using Method 6, a judge could indicate nonlinear cue utilization since values or ranges of values of each cue were rated separately. But overall cue weights were assessed by the same procedure used for Method 1. For the final method a special nine-point rating scale was devised to allow a judge to express information about nonlinear cue utilization and cue weight with a single rating. Judges used this scale to rate values of each cue as if this were the only information known about a candidate (specific representative values, rather than ranges, were chosen for the numerical cues). The points on the nine-point scale were described in such a way that they reflected the extent to which a given value of one cue was evaluated in light of not only other values of the same cue, but also in light of information about other cues. Although the judge evaluated only one cue at a time, he was thus able to indicate information about the relative weight of a cue with respect to the other cues used in the task. For example, a rating of 9 would indicate that the cue value being rated was both high enough and important enough to be treated as a "critical" value and the applicant would receive a high rating regardless of the values other cues might have. An intermediate rating of 5 would be given to the value of a cue if it would have neither a positive nor a negative influence on a judge's ratings of a candidate. A rating of 7 indicated a cue value that would play a relatively important positive role in determining the overall rating for an applicant.

\section{Measures}

The subjective estimates of cue weights were used to generate predicted ratings for each candidate. For judges using one of the first five rating methods, the subjective weights were used as if they were beta weights and predicted ratings obtained by multiplying subjective weights by the standardized values of the cues. Predicted ratings were obtained for Method 6 by summing weighted subjective ratings of the cue values. The process was similar for those judges using Method 7, except that separate cue weights were not given by these judges. The ratings from the nine-point scale corresponding to the cue values for each candidate were thus simply summed to obtain predicted ratings.

These predictions were then correlated with the actual ratings of the judges. The squared values of these correlations $\left(r^{2}\right.$ in Table 1$)$ represent the variance accounted for by a linear model based on subjective weights. 


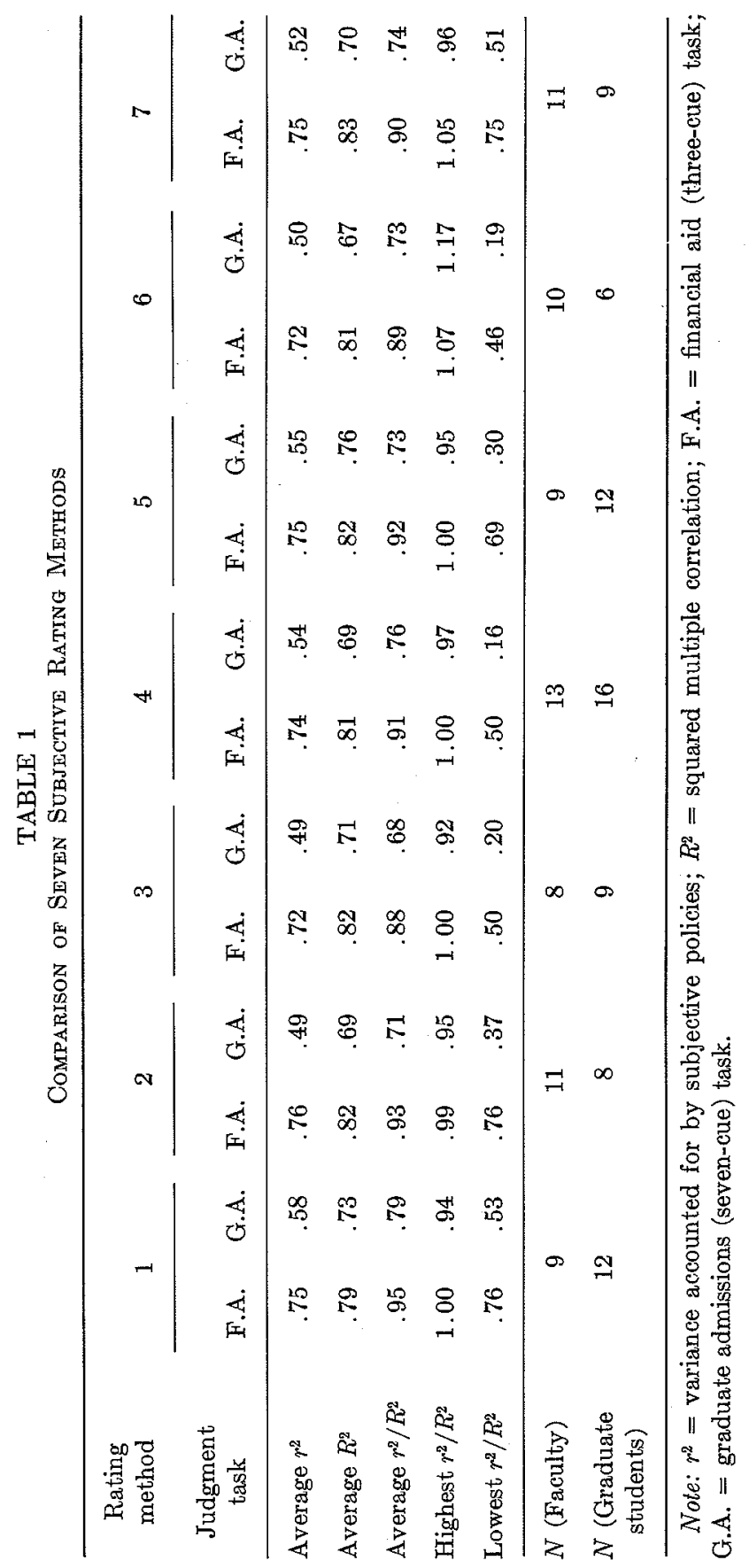


(These correlations are invariant for any multiplicative transformation of the subjective weights.)

The squared $r$ 's can be compared across rating methods. They also can be compared to the variance accounted for by the best linear model, derived from a multiple regression analysis ( $R^{2}$ in Table 1$)$. In addition to average values of $r^{2}$ and $R^{2}$, averages of the ratio $r^{2} / R^{2}$ are also presented in Table 1. This ratio represents the proportion of the variance accounted for by using subjective weights compared to the maximum proportion of the variance that can be accounted for by a linear model. This ratio could exceed 1.0 only for Methods 6 and 7 , and then only if the judge accurately reports nonlinear cue utilization.

As another basis for comparing methods, a set of predicted ratings was derived for each task assuming equal weights on the cues. Such a policy serves as a "baseline" comparison policy in that an outside observer who wished to predict the judgments of a randomly chosen judge might well use such a policy if he did not have access to any other information about the judge (see also Dawes, 1972). The variance accounted for by predictions based on this policy is not a lower limit for the variance that might be accounted for by an inaccurate subjective policy.

As with ratings derived from subjective weights, predicted ratings based on the application of equal weights to standardized cue values were correlated with each judge's actual ratings and the squared values of these correlations were then averaged. The average squared baseline correlation thus obtained was .62 for the financial aid task and .39 for the graduate admissions task.

\section{RESULTS}

The effect of order of presentation of the two tasks was not significant and the two orders are combined in the following analyses.

An analysis of variance on the values of $R^{2}$, with the task factor (financial aid vs graduate admissions) treated as a repeated measure, shows that the judges in the different rating conditions did not differ significantly in terms of the linear predictability of their judgments $(F(6,219)=.60$, n.s. $)$.

The difference between the two tasks is, however, quite significant $(F(1,129)=104.64, p<.01)$. And, while the main effect for Judges (faculty vs graduate students) was not significant $(F(1,129)=.75$, n.s.), the Judges-by-Task interaction was $(F(1,129)=4.11, p<.05)$.

The main effect for Tasks is reflected in the values of $R^{2}$, which are .82 for the financial aid (three-cue) task and .71 for the graduate admission (seven-cue) task. The significant Judges-by-Task interaction occurs because of a slightly higher $R^{2}$ for graduate students on the finan- 
cial aid task (.83 vs .80 for faculty), a difference that is not present in the graduate admissions task (average $R^{2}$ of .71 for both groups).

The seven rating methods do not differ significantly $(F(6,129)=.72$, n.s.) when squared correlations between predicted and actual ratings $\left(r^{2}\right)$ are compared. The difference between tasks, however, is significant $(F(1,129)=219.96, p<.01)$. The average $r^{2}$ for the financial aid task is .74 and for the graduate admissions task, .53 .

The only other significant effect for $r^{2}$ is (as above) the Judges-byTasks interaction $(F(1,129)=7.32, p<.01)$. The average for the graduate students is once more higher than that of the faculty for the financial aid task (.76 and .72, respectively), but the graduate students fall below the faculty on the graduate admissions task (values of .51 and $.54)$.

An analysis of variance was also done for the $r^{2} / R^{2}$ ratio, since this should be a somewhat more sensitive test of the differences between the rating methods. Individual differences in the values of $R^{2}$ are controlled and the ratio more directly assesses the degree to which predicted ratings made from subjective weights approach the best linear predictions from multiple regression. But, the results for the analysis of $r^{2} / R^{2}$ are essentially the same as for the individual measures; in particular, the rating methods do not differ significantly $(F(1,129)=1.19, p>.25)$, while the Tasks-by-Judges interaction is again significant $\left(F^{\prime}(1,129)=4.13, p<\right.$ $.05)$. The interaction occurs because the average value of $r^{2} / R^{2}$ is lower for graduate students than for faculty for the graduate admissions task (.71 vs .76), while the values are comparable for the two groups for the financial aid task (approximately .91).

The results for the highly significant task differences may be summarized by noting differences between the tasks in terms of variance accounted for. For each of the three sets of weights (statistical, subjective, and equal) there is less variance accounted for in the graduate admissions task than in the financial aid task. For the graduate admissions task optimal linear weights account for an average of $71 \%$ of the variance, subjective weights for $53 \%$, and equal or baseline weights for $39 \%$. The corresponding figures for the financial aid task are 82, 74, and $62 \%$. Variance accounted for by subjective weights is intermediate in both cases, but proportionately closer to the maximum variance accounted for by a linear model for the financial aid task.

Multiple correlations are shown in Table 1 because they represent an absolute upper limit for variance explained by linear weights in the sample of profiles judged, but the multiple correlations should show some shrinkage if the regression weights are applied to a different sample. The amount of shrinkage depends upon the number of cues and the 
number of profiles in the sample. Wherry's formula (Wherry, 1931; see also Herzberg (1969)) was used to compute $R_{s}{ }^{2}$, the squared multiple correlation corrected for shrinkage. For each subject, the value of $R^{2}$ was transformed according to the formula

$$
R_{s}{ }^{2}=1-[(N-1) /(N-n-1)]\left(1-R^{2}\right),
$$

where $N$ is the number of judgments in a task (sample size) and $n$ is the number of cues (independent variables).

The results of analyses of variance of $R_{s}{ }^{2}$ and of $r^{2} / R_{s}{ }^{2}$ are quite similar to the results of analyses of $R^{2}$ and $r^{2} / R^{2}$. The rating methods do not differ significantly, but the difference between tasks is significant at well beyond the .001 level. However, the Tasks-by-Subjects interactions are not quite significant at the .05 level (for $R_{s}{ }^{2}, F=3.71$ and for $r^{2} / R_{s}{ }^{2}$, $F=3.66$; in both cases $d f=1,129$ and $.10>p>.05$ ). Values of $R_{s}{ }^{2}$ and $r^{2} / R_{s}{ }^{2}$ averaged across the seven rating methods are included in Table 2, as are values involving $r^{2}, R^{2}$, and $r_{0}{ }^{2}$ (the variance accounted for by the baseline policy described above).

For rating methods $1-5, R^{2}$ represents the upper limit for $r^{2}$ since the predicted ratings are arrived at by a linear combination of the cue values. As noted, rating Methods 6 and 7 allowed judges to express their subjective weights in more complex ways and the resulting predicted ratings include information about nonlinear cue utilization.

As can be seen by examining the highest $r^{2} / R^{2}$ values in Table 1 , there were judges for whom the inclusion of this information resulted in predicted ratings that correlated highly with actual ratings, so that the variance accounted for by the correlation between actual and predicted ratings exceeded that variance accounted for by the best linear combination of the cues. For the financial aid task there were three judges (out of 16) using Method 6, and two judges (out of 20) using Method 7,

TABLE 2

Comparison of the Two Tasks, Average Values Over Seven Methods

\begin{tabular}{ccccccccc}
\hline Task & $r^{2}$ & $r_{b}{ }^{2}$ & $R^{2}$ & $R_{s}{ }^{2}$ & $r^{2} / R^{2}$ & $r_{b}{ }^{2} / R^{2}$ & $r^{2} / R_{s}{ }^{2}$ & $r_{b}{ }^{2} / R_{s}{ }^{2}$ \\
\hline $\begin{array}{c}\text { Financial aid } \\
\quad \text { three cues; } n=71)\end{array}$ & .74 & .62 & .82 & .80 & .91 & .76 & .93 & .71 \\
$\begin{array}{c}\text { Graduate admissions } \\
\quad(\text { seven cues; } n=72)\end{array}$ & .53 & .39 & .71 & .66 & .74 & .55 & .80 & .59 \\
\hline
\end{tabular}

Note: $r^{2}=$ variance accounted for by subjective policies; $r_{b}{ }^{2}=$ variance accounted for by "baseline". policy of equal cue weights; $R^{2}=$ squared multiple correlation; $R_{s}{ }^{2}=R^{2}$ corrected for shrinkage. 
for whom $r^{2}$ exceeded $R^{2}$. For the graduate admissions task, $r^{2}$ exceeded $R^{2}$ for one judge using Method 6 .

The nine-point rating scale used for Method 7 was defined in such a way that the end points represent extreme judgments that are noncompensatory in nature. A subject giving a rating of 9 to a 4.0 GPA is indicating he would probably ignore all other information in arriving at an overall judgment. Thus a transformation of the scale values that gives more weight to extreme ratings might be expected to be appropriate for at least some judges.

To examine the effect of such transformations, deviations of each judge's ratings from the midpoint value of 4 were squared and cubed (retaining the sign) and these transformed ratings used to produce two new sets of overall judgments. Use of one or both of these sets of judgments based on transformed ratings did, in fact, result in higher proportions of variance accounted for in some cases. If the set of judgments yielding the highest $r^{2}$ for each judge is chosen, then $r^{2}$ exceeds $R^{2}$ for four (instead of two) judges on the financial aid task and the lowest values of $r^{2} / R^{2}$ are .82 and .60 for the financial aid and graduate admissions tasks, respectively. Also, the average value for $r^{2}$ increases from .75 to .79 for the financial aid task and from .52 to .55 for the graduate admissions task.

Finally, Methods 6 and 7 provide the necessary information for subjective rescaling of the cue values (see Doherty \& Keeley, 1972). To examine the usefulness of subjective descriptions of cue utilization, multiple regression analyses were performed for each of these judges with subjective ratings of cue values as the values of the independent variables. The average $R^{2}$ using these subjectively rescaled cues for judges who had used Method 6 was .83 for the financial aid task and .66 for the graduate admissions task; for Method 7 the respective values were .83 and .70 . These values differ little from the average squared multiple correlations derived from the original analyses, as can be seen by comparison with the figures given in Table 1 , but this result may be due, in part, to the nature of the tasks, a point discussed below.

\section{DISCUSSION}

The two important conclusions to be drawn from these results are that (1) the rating methods did not differ, and (2) subjective policy descriptions corresponded fairly closely to statistical policy descriptions. But these conclusions should be tempered by two qualifications that are related to the nature of the two judgmental tasks and the use of group averages to assess differences between methods. Each of these conclusions 
will be discussed in turn and the relevance of these qualifications will be indicated.

\section{Differences Between Rating Methods}

The fact that the rating methods chosen to obtain subjective policy descriptions did not differ significantly was surprising. Because these methods included two complex approaches to assessing both cue weights and nonlinear cue utilization, as well as one method that could be described as rather crude (rating the number of times a cue was "influential"), differences were expected. These results indicate, therefore, that the often used method of asking a subject to divide 100 points appears to be as good as any other method for obtaining subjective weights. This generalization is likely to hold for similar tasks if group averages are used. Both the financial aid and graduate admissions tasks employed cues that most judges probably felt were positively and monotonically related to the judgmental criterion. It is likely that there would be differences between rating methods with tasks involving markedly nonlinear, configural, or negative cue-criterion relationships. That the utilization of information about nonlinear cue-criterion relationships in the present study accounted for more variance than the best linear model for a few judges using Methods 6 and 7 is evidence for this point.

The results for Methods 6 and 7 also suggest that the use of group averages may have obscured important individual differences. The use of such relatively complex methods for assessing subjective policies is apparently appropriate for some, but not all, subjects. The rating method and the analysis need to be fitted to the judge. The choice of a subjective rating method would depend upon both the complexity of the judge's policy and the judge's ability to describe the policy. The ability to describe a policy should improve with practice and appropriate training. The problem of choosing the right rating method and the possibility of training judges to use these methods will be referred to again after the accuracy of the subjective policies is examined.

\section{Accuracy of Subjective Policies}

As noted in the introduction, accuracy of subjective policies has been defined in terms of the correspondence between subjective and statistical policies. The question is how much variance is accounted for by the use of subjective policy descriptions when compared to the maximum variance accounted for by a linear model? When examined from this perspective the subjective policy descriptions are reasonably accurate, particularly for the three-cue financial aid task. For this task subjective policy descriptions account for fully $91 \%$ of the maximum linear vari- 
ance. For the graduate admissions task $74 \%$ of the linear variance is accounted for by subjective weights. But the multiple correlation capitalizes on chance for any given set of data, and a more meaningful comparison is with the average squared multiple correlations after the correction for shrinkage. For the financial aid task and the graduate admissions task, subjective policy descriptions account for 93 and $80 \%$ of the estimated linear variance, respectively.

As noted earlier, Summers et al. found that the best linear model accounted for $20 \%$ more variance than a model based on subjective weights. Their study involved only a four-cue task, but the task might have been difficult for the undergraduate judges, since it was one with which they had no previous experience. In the present study both graduate student and faculty judges were presented with a decision problem and cues with which they were quite familiar. The task was then more meaningful and realistic, even though the actual profiles were hypothetical.

An explanation consistent with these findings is that as the difficulty of the task increases, the judge becomes both less consistent in the application of his judgmental policy as well as less able to describe it.

The Tasks-by-Subjects interactions are probably due to differences in task complexity for the two groups of judges, but there is no way to know, in the context of the present study, whether this is due to differences in ability between the two groups of judges or to group differences in policies used for the two tasks. Because of substantive differences between the two tasks employed in the present study, evidence for the source of this interaction would have to come from a comparison of these groups on other tasks.

The problem of group averages obscuring individual differences is also relevant to comments on the accuracy of subjective policy descriptions. The performance of the models based on subjective weights would have been higher if the scaling method best suited to each judge's policy and abilities had been chosen.

One final comparison that bears on the accuracy of the subjective weights is with the "baseline" policy of equal weights on all cues. Such a policy is arbitrary and, as noted earlier, it would be possible for a judge to specify a set of subjective weights that corresponds less closely to the statistical weights than the policy of equal weights on the cues. But the baseline correlations do provide a useful comparison if this qualification is kept in mind. Dawes (1972) has shown that, because of the robustness of the linear model, a policy of equal weights will often predict an external criterion more accurately than the subjects' actual judgments. 
While an examination of the data does not show any consistent pattern of cue utilization across judges, the average subject was clearly able to give a set of subjective weights that yielded more accurate predictions of judgments than did the arbitrary policy of equal weights. The use of the judges' descriptions of their own policies results in an increase of variance accounted for over the baseline policy of $12 \%$ for the financial aid task and $14 \%$ for the graduate admission task.

\section{Conclusions}

The seven methods used to assess subjective policies did not differ in their accuracy, and subjective policy descriptions were in reasonable accord with statistical policy descriptions. These conclusions hold for both faculty members and graduate students and for both tasks, but the correspondence between subjective and statistical policies were greatest for the three-cue task.

Further research into the substantive questions considered in this paper is warranted, and could profitably address itself to the role of both individual differences and task differences. The method to be preferred for assessing subjective weights might depend on an estimate by the judge of the complexity of his subjective policy and upon an independent measure of his understanding of the policy. If complex tasks were chosen that involved a large number of cues, negatively weighted cues, or nonmonotonic cue-criterion relationships, then differences between rating methods might emerge.

\section{REFERENCES}

Bloop, M. R. The validity of importance. Journal of applied psychology, 1971, 55, $487-488$.

Darlington, R. B. Multiple regression in psychological practice and research. Psychological bulletin, 1968, 69, 161-182.

Domerty, M. E., \& Keelex, S. M. A note on the use of subjective predictors in regression analysis for policy capturing. Journal of applied psychology, 1972, 56, $277-278$.

Dawes, R. M. Slitting the decision maker's throat with Occam's Razor: The superiority of random linear models to real judges. Oregon research institute research bulletin, 1972, 12, 13.

Dudycha, L. W., \& NAYLOR, J. C. Characteristics of the human inference process in complex choice behavior situations. Organizational behavior and human performance, 1966, 1, 110-128.

EinhorN, H. J. The use of nonlinear, noncompensatory models in decision making. Psychological bulletin, 1970, 73, 221-230.

EinhorN, H. J. Use of nonlinear, noncompensatory models as a function of task and amount of information. Organizational behavior and human performance, $1971,6,1-27$.

Goldberg, L. R. Man versus model of man: A rationale, plus some evidence, for a 
method of improving on clinical inferences. Psychological bulletin, 1970, 73, $422-432$.

Goldberg, L. R. Five models of clinical judgment: An empirical comparison between linear and nonlinear representations of the human inference process. Organizational behavior and human performance, 1971, 6, 458-479.

Hammond, K. R., \& Summers, D. A. Cognitive control. Psychological review, 1972, $79,58-67$.

Herzberg, P. A. The parameters of cross-validation. Psychometrika, 1969, 34 (2, Pt. 2).

Hoffman, P. J. The paramorphic representation of clinical judgment. Psychological bulletin, 1960, 57, 116-131.

Hoffman, P. J., \& Blanchard, W. A. A study of the effects of varying amounts of predictor information on judgment. Oregon research institute research bulletin, 1961.

Permut, S. E. Cue utilization patterns in student-faculty evaluation. Journal of psychology, 1973, 83, 41-48.

Slovic, P. Analyzing the expert judge: A descriptive study of a stockbroker's decision processes. Journal of applied psychology, 1969, 53, 255-263.

Slovic, P., Fleissner, D., \& Bauman, W. S. Analyzing the use of information in investment decision making: A methodological proposal. Journal of business, $1972,45,283-301$.

Slovic, P., \& Lichtenstein, S. C. Comparison of Bayesian and regression approaches to the study of information processing in judgment. Organizational behavior and human performance, 1971, 6, 649-744; also, In L. Rappoport \& D. A. Summers (Eds.), Human judgment and social interaction. New York: Holt, Rinehart \& Winston, 1973.

Summers, D. A., Taliaferro, J. D., \& Fletcher, D. J. Subjective vs objective description of judgment policy. Psychonomic science, 1970, 18, 249-250.

Torgerson, W. S. Theory and methods of scaling. New York: Wiley, 1958.

WHERRY, R. J. A new formula for predicting the shrinkage of the coefficient of multiple correlation. The annals of mathematical statistics, 1931, 2, 440-457.

Received: May 7, 1973 